unter anderem seine Kommentierungen auch dieser Regelungen auf den neuesten Stand gebracht. Dies gilt ebenso für Gerhard Robbers, der auf seinem Spezialgebiet, dem Staatskirchenrecht, die entsprechenden Bestimmungen weiterhin kommentiert. Die meisten der früheren Mitarbeiter haben den Themenbereich gewechselt, so unter anderem der Mitherausgeber Lars Brocker, der dieses Mal - entsprechend seiner neuen Position - den Abschnitt „Die Rechtsprechung“ übernommen hat.

Besonders lesenswert sind die Erläuterungen zum Verfassungsgerichtshof. Im Gegensatz zum Vorgängerkommentar haben nicht Richter des Oberverwaltungsgerichtes beziehungsweise Mitglieder des Verfassungsgerichtshofes diese wichtigen Bestimmungen bearbeitet, sondern eine Persönlichkeit, die vor dem Richtertisch steht. Als zuständiger Abteilungsleiter und Vertreter des öffentlichen Interesses hat Jutzi über Jahre in vielen Prozessen wertvolle Erfahrungen gesammelt und ist auch durch eine Vielzahl von wissenschaftlichen Veröffentlichungen in diesem Bereich anerkannt. Seine umfassenden Erläuterungen zeigen zudem, dass ihm zudem seine langjährige Lehrtätigkeit auf dem Gebiet des Landesverfassungsrechts einschließlich des Verfassungsprozessrechts zugute kommt. Dass die Kommentatoren anscheinend bis zur letzten Minute ihre Manuskripte auf den neuesten Stand gebracht haben, kann zum Beispiel an der Kommentierung des Bonner Staatsrechtslehrers Klaus F. Gärditz verdeutlicht werden: Er hat bei seinen Erläuterungen der Bestimmungen über die Regierungsbildung einen Aufsatz, der Ende 2013 erschienen ist, noch berücksichtigt.

Die Neukonzeption dürfte den Bedürfnissen von Wissenschaft und Praxis voll entsprechen. Es ist zu hoffen, dass trotz des hohen Preises die Entscheidungsträger das Werk immer dann zu Rate ziehen werden, wenn kleine und große Probleme des rheinland-pfälzischen Staats- und Verfassungsrecht zur Entscheidung anstehen. Da es in anderen Bundesländern in vielen Bereichen ähnliche Regelungen gibt, sind die Kommentierungen jedoch nicht nur für Rheinland-Pfalz von eminent praktischer Bedeutung

Richard Ley

\title{
Austausch von Landtagsabgeordneten: breite Bestandsaufnahme, komplexe Berechnun- gen, forsches Urteil
}

Heinsohn, Till: Mitgliederfluktuation in den Parlamenten der deutschen Bundesländer. Eine Bestandsaufnahme und Ursachenforschung (Reihe: Policy-Forschung und Vergleichende Regierungslehre, Band 17), LIT Verlag Dr. W. Hopf, Berlin 2014, 289 Seiten, € 39,90.

Mit der Frage nach Umfang, Gründen und Ursachen parlamentarischer Mitgliederfluktuation in deutschen Landesparlamenten zwischen 1946 und 2012 führt Heinsohn seine Leser wissenschaftlich exakt durch seine von Markus Freitag und Marc Debus begleitete und von der DFG geförderte Dissertation an der Universität Bern. Begrifflich sauber ersetzt er den gängigen Begriff der Elitenzirkulation durch parlamentarische Mitgliederfluktuation. In einer Makro- und Mikroebene vereinenden Analyse bildet er „das Ausmaß und die Variation parlamentarischer Mitgliederfluktuation in den deutschen Bundesländern zwischen 1946 und 2012 umfassend über die Untersuchungseinheiten hinweg vergleichbar ab“ (S. 73). 
Seine umfangreiche Literaturauswahl folgt der Policy-Forschung und der vergleichenden Regierungslehre. Arbeiten zu Parteien und Fraktionen sowie Wahlrecht werden kaum einbezogen. Aus dem internationalen Vergleich heraus hält er den Austausch von einem Drittel der Abgeordneten für wünschenswert, da damit sowohl Erneuerung als auch Arbeitsfähigkeit des Parlaments bedient wird, wobei der Maßstab für dieses Urteil weder parlamentarismustheoretisch noch empirisch begründet wird.

Da eine umfassende Bestandsaufnahme fehlt, musste in einem ersten Schritt ein umfassendes Bild der aggregierten Veränderungen am Wahltag (ordentlichen) und denen im Verlauf der Wahlperiode (außerordentlichen) in den deutschen Bundesländern zwischen 1946 und 2012 erstellt werden. Der umfangreiche Datensatz aus den elf alten Bundesländern in bis zu dreizehn Wahlperioden und fünf neuen Bundesländern mit bis zu sechs Wahlperioden, getrennt nach ordentlicher und außerordentlicher Fluktuation weist mit 50 Prozent eine so hohe Rate aus, dass Heinsohn von äußerst instabilen Parlamenten spricht (S. 24). Die dabei festgestellten großen Niveauunterschiede führen zu der Frage: „Welche kontextuellen und personengebundenen Faktoren beeinflussen die individuelle Wahrscheinlichkeit eines Rücktritts, eines Kandidaturverzichts oder einer Abwahl aus dem Parlament“ (S. 16)?

Heinsohn erarbeitet anhand der Kriterien: Wahlsystem, Listenform, Dauer der Wahlperiode, Inkompatibilität, Institutionalisierung, Professionalisierung, Größenveränderung der Parlamente, Volatilität und Aufstiegsmöglichkeiten Hypothesen und Wirkungsrichtungen der ordentlichen Fluktuation. Diese aus dem Studium der erklärenden Forschung abgeleiteten Variablen verbindet er in zehn Modellen, mit denen er seinen umfangreichen Datensatz auswertet. Für die individuelle Rücktrittswahrscheinlichkeit ist das Zusammenspiel von kontextuellen - Verweildauer, zusätzliches Regierungsamt, Pensionsansprüche und die Höhe der finanziellen Entschädigung - verbunden mit den personengebundenen Faktoren wie Alter, Familienstand, Bildungsabschluss und offizielles Amt im Parlament relevant. Die aus den Handbüchern der Landesparlamente schwerer zu ermittelnden Daten der außerordentlichen Fluktuation zwischen 1990 und 2012 werden nach Direkt- oder Listenmandat, der Verweildauer, Regierungsamt, Pensionsanspruch, Entschädigung, Parteipopularität und Mitgliederzahl der Parteien, Geschlecht, Alter, Familienstand, Doktortitel, Beruf, Dauer der Parteimitgliedschaft sowie Parlamentsamt mit den Hypothesen konfrontiert, um so die unterschiedlichen Entwicklungen der Bundesländer darzustellen und zu erklären.

Im auch methodisch rückschließenden Forschungsausblick empfiehlt er zwischen unterschiedlichen Austrittsformen zu differenzieren und zu prüfen, ob die Handlungsfähigkeit durch Austausch der Entscheidungsträger so gefährdet ist, dass der Einfluss von Wählern und ihre Chance, Interessen einzubringen, eingeschränkt werden müsste.

Die „Vermutung, dass sich die hohen Fluktuationsraten nicht gerade förderlich auf die Handlungsfähigkeit der subnationalen Parlamente auswirken und zumindest in Teilen zu einem Verschwimmen von politischer Verantwortlichkeiten beitragen“, führt ihn zu der „Frage nach den Stellschrauben, die mit Blick auf eine aktive politische Einflussnahme in den Bundesländerparlamenten bestehen“ (S. 235). Ziel ist dabei, „die Möglichkeit einer aktiven politischen Einflussnahme abzuwägen“ (S. 236). Danach sind die Dauer der Wahlperiode, Institutionalisierung und Professionalisierung des Parlaments als stimulierende Anreize möglich, da wechselnde Wählerpräferenzen und damit einhergehende Veränderungen der Parlamentsgröße durch Überhang- und Ausgleichsmandate nicht nur demokratietheoretisch unbeeinflussbar bleiben sollten. 
Wie vorgeschlagen, sollte geprüft werden, ob die Handlungsfähigkeit der Parlamente gefährdet ist (S. 239). Heinsohns Untersuchung zeigt jedenfalls, dass das in Hamburg, Bremen und im Saarland praktizierte Verhältniswahlrecht mit einer höheren Fluktuation einhergeht. Diese wird in den beiden Stadtstaaten zudem durch Inkompatibilitätsregelungen und geringere Entschädigung befördert. Es ist danach zu fragen, wieweit die Arbeitslast bei größeren Wahlkreisen und Kompetenzerweiterung der Parlamente durch bessere Ausstattung der Landtagsabgeordneten ausgeglichen werden sollte. Dies würde allerdings die Chancen der Wahlkreiskonkurrenten verschlechtern.

Der flüssig geschriebene Text regt zum Nachdenken und zu weiterer Forschung an, weil er auf die Unterschiede zwischen den Bundesländern und damit auf die komplexen Zusammenhänge hinweist. So zeigt die Untersuchung der Veränderungen im Abgeordnetenhaus von Berlin, dass (1) die Formen des personalisierten Wahlrechts doch signifikante Unterschiede produzieren; (2) Inkompatibilität auch mit dem Wechsel in Bundestag und Bundesregierung einhergeht, zudem für Staatssekretäre und kommunale Ämter ebenfalls relevant ist und deshalb Kommunalverfassungen zu berücksichtigen sind; (3) Veränderungsraten der Fraktionen sich nicht nur durch Wählerentscheid, sondern auch durch die Nominierungsbedingungen (Landes- oder Bezirkslisten, Mitglieder- oder Delegiertenversammlungen) und die Mitgliederzahl erklären lassen. ${ }^{1}$

Um einen „Ausreißer“ reduziert, liefert der Vergleich zwischen den Bundesländern praxisrelevante Hinweise zur Stabilisierung der Landesparlamente und relativiert das Urteil „instabiler“ Verhältnisse.

Ingrid Reichart-Dreyer

1 Vgl. Ingrid Reichart-Dreyer, Wer wählt die Abgeordneten aus? Einfluss von Wählern und Parteien auf die Zusammensetzung des Abgeordnetenhauses von Berlin 1963 bis 2011, in: ZParl, 43. Jg. (2012) H. 1, S. $36-53$.

\section{Politische Theorie und Rechtsphilosophie: Chance auf Verbindung wenig genutzt}

Jovanovic, Miodrag A. und Bojan Spaić (Hrsg.): Jurisprudence and Political Philosophy in the 21st Century. Reassessing Legacies (Central and Eastern European Forum for Legal, Political, and Social Theory, 2nd Yearbook), Peter Lang, Frankfurt am Main u.a. 2012, 167 Seiten, $€ 43,95$.

Der Titel verspricht ein ehrgeiziges Unterfangen: Die philosophischen Bestände rechtlicher und politischer Ordnungsentwürfe sollen neu evaluiert werden. Da es sich um ein Jahrbuch des jungen Forums mittelosteuropäischer Forscher handelt („Central Eastern European Forum for Legal, Political, and Social Theory“), liegt es nahe, den Grund für ein solches „reassessing" mit der spezifischen Perspektive Mittel- und Osteuropas in Verbindung zu bringen. Und die beiden Herausgeber befördern in ihrer Einleitung eine solche Erwartung, wird doch die spezifische Perspektive des „Ostens“ explizit in Anspruch genommen, um sie als Grundlage erfolgter und erfolgreicher Traditionsbildung des Forums darzustellen. Dass 